\title{
Does having both ADHD and irritability symptoms in childhood predict mental health outcomes in adolescence?
}

\author{
By Dr. Jessica Edwards
}

Attention-deficit/hyperactivity disorder (ADHD)

symptoms combined with high levels of irritability during childhood is a significant predictor of subsequent mental health problems and suicidality in adolescence, according to findings from a new study. To draw this conclusion, Cedric Galera and colleagues followed $>1,400$ children from the general population who were enrolled in the Québec Longitudinal Study of Child Development.

First, they identified the developmental trajectories of childhood ADHD and irritability symptoms between the ages of 6 and 12 years. They then analysed whether these trajectories were associated with psychiatric symptoms, functional impairment and suicidal ideation or attempts between 13 and 17 years of age.

The researchers identified distinct childhood developmental profiles, as follows: absent/very low ADHD and irritability (which affected $67 \%$ of the cohort), moderately high irritability and low ADHD (11\%), moderately high ADHD and low irritability (14\%), and both high ADHD and high irritability (8\%). During adolescence, participants with high childhood ADHD and irritability had higher levels of ADHD symptoms, externalizing and internalizing symptoms, and functional impairment, compared to all three other groups. They were also more likely to experience suicidality than the first two groups.

Galera et al. explain that more children from lower socioeconomic backgrounds were lost to follow up, so their study sample likely represents young people at lower risk and the observed associations might be underestimates. However, pending replication of these findings, the researchers believe that there is still good reason for clinicians to consider irritability symptoms when diagnosing and assessing ADHD. Further studies are now warranted to determine whether decreasing childhood irritability might improve adolescent mental health and suicidal outcomes in children with ADHD.

\section{Referring to:}

Galera, C., Orri, M., Vergunst, F., Melchior, M., Van der Waeden, J., Bouvard, M.P., Collet, O., Boivin, M., Tremblay, R.E. \& Côté, S.M. (2020), Developmental profiles of childhood attentiondeficit/hyperactivity disorder and irritability: association with adolescent mental health, functional impairment, and suicidal outcomes. J. Child Psychol. Psychiatr. doi: 10.1111/jcpp.13270.

\section{Glossary:}

Internalizing symptoms: emotional symptoms which tend to be experienced internally. In this study the internalizing symptoms assessed included social phobia, generalized anxiety, and depression symptoms.

Externalizing symptoms: maladaptive behaviours which are directed externally towards the environment. In this study the externalizing symptoms assessed included oppositional defiant disorder, conduct disorder, psychopathy, delinquency and contact with police, and aggression symptoms.

Functional impairment: limitations to daily life and usual roles. For example, difficulty completing school-work or socializing with friends.

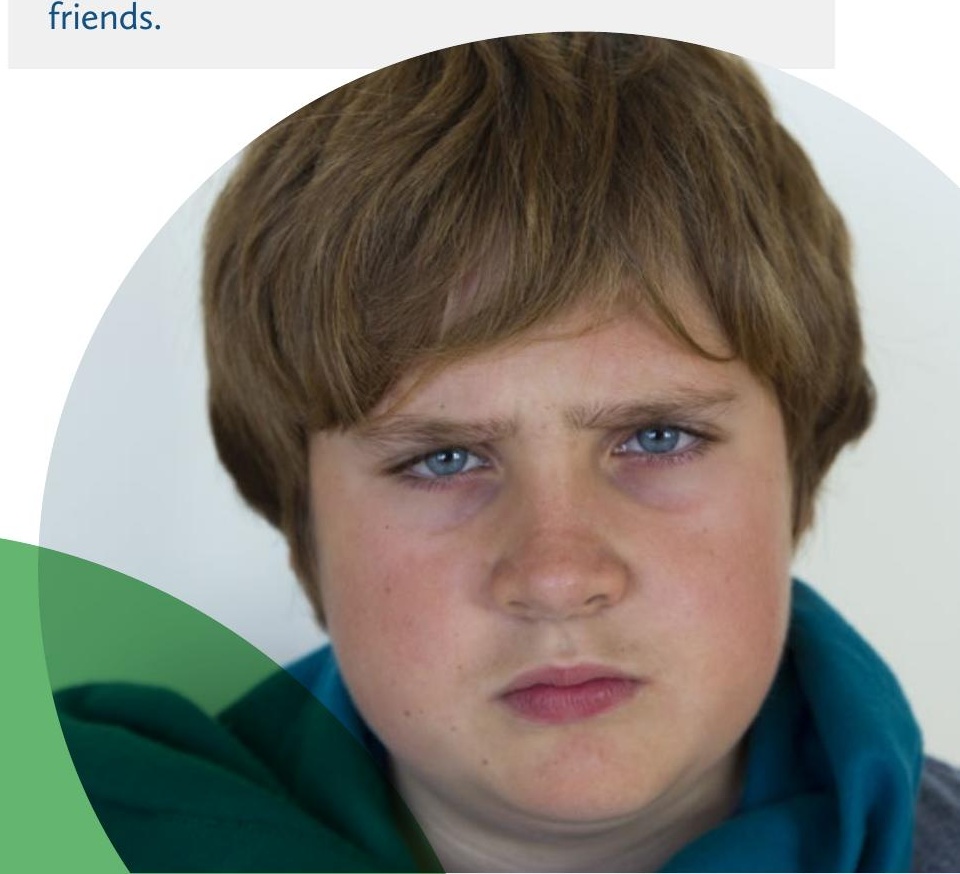

